Archived version from NCDOCKS Institutional Repository http://libres.uncg.edu/ir/asu/

Pine, J. C. (2004). Research needs to support the emergency manager of the future. Journal of Homeland Security and Emergency Management: Vol. 1: Iss. 1, Article 3.

DOI: 10.2202/1547-7355.1012 (ISSN: 1547-7355) Published by De Gruyter Company. This version of the record is available from http://www.degruyter.com/view/j/jhsem

\title{
Research Needs to Support the Emergency Manager of the Future John C. Pine
}

\begin{abstract}
Changes in technology have dramatically transformed emergency management operations from interagency communications, to decision support systems, agency hazards analysis, and training. Dramatic advances in technology have and will continue to enhance elements of decision-making by those involved in emergency management. This paper outlines future research needs associated with the application of technology to emergency management and outlines operational issues associated with the application of technology to the emergency management system. Emergency management research will need to support developments in the technology and its impacts on the design and support of emergency management operations. Interdisciplinary research must bring new technology to traditional emergency management agencies and to integrate public, private and non-profit agencies in the emergency management process. Enhancing partnerships with other agencies allows for a broader approach to addressing emergency management issues.
\end{abstract}

\section{Summary}

The emergence of the intelligent city in the latter part of the 20th Century and the beginning of the 21st Century has dramatically transformed our understanding of and capacity to cope with disasters (Stalberg). Changes in technology have dramatically transformed emergency management operations from interagency communications, decision support systems, agency hazards analysis, and training. Dramatic advances in 
technology have and will continue to enhance elements of decision-making by those involved in emergency management. Mobile wireless networks are now serving as an element of communications and offers access to a variety of resources that were recently

only available at a very high cost. As a result of a greater interconnectedness of public, private, and non-profit agencies, traditional organization boundaries will undergo significant change. This paper outlines future research needs associated with the application of technology to emergency management and outlines operational issues associated with the application of technology to the emergency management system. Emergency management research will need to support developments in the technology and its impacts on the design and support of emergency management operations.

\section{Developments in Technology}

The technology infrastructure brings a variety of new applications to emergency management. The technology involves powerful portable computers, digital telephones, satellite imaging and sensors, environmental sensors, networks, both line and wire-less. It includes remote sensing for data collection and geo-positioning devices for pinpointing locations (shelters, incidents, facilities, schools, hospitals, nursing homes, schools) and wire line and wireless networks). Future research in the technology infrastructure will continue to increase the capacity of the emergency management system. This infrastructure provides the technological basis for communications and program applications.

Continued developments in acquiring many new data sources, program applications including more sophisticated hazard modeling capabilities, and means of linking our systems require the knowledge and skills of many disciplines. Unfortunately, the specialists become more isolated from one another and from the emergency management community. The research community needs to work in an interdisciplinary manner to address the complex problems associated with natural and man-made hazards and form partnerships with the emergency management community.

Information and Decision Support Systems:

The infrastructure also includes computer program applications and data used in these programs. Current program applications include information management (CAMEO), modeling software (HPAC \& HAZUS-MH, \& ALOHA), and telecommunication warning systems (I-Notification) and support a variety of emergency management functions. Computer programs will make it easier for managers to communicate with one another, access data, and analyze information. Geo-positioning systems (GPS) and geographic information systems (GIS) will merge current database tables to graphic intelligent maps. Technology for storm prediction and tracking allows for more effective preparedness activities including sheltering, evacuation, and damage assessment. Programs for automated forecasts, modeling, warning, evacuation modeling, and digital libraries can be used for training, planning and mitigation activities 
and are based on databases that in the past were not available to emergency managers.

\section{Modeling}

In the real world, a disaster is a complex phenomenon (Drager, 1993). The wind velocity, the surface roughness, air temperature, and surface feature all contribute to the effects of natural events and chemical dispersion. With this inherent complexity, models which attempt to replicate a real world system must make simplifying assumptions. It follows that attempts to exactly model a natural or man-made phenomenon via a mathematical algorithm must, by the limitations of the technology, be only an approximation. The accuracy of the model's estimation of an event is determined by both the assumptions contained within the model and by the assumptions made by the user as the data sets are prepared for modeling or inputs are entered in the user interface with the model. Major developments will need to continue to enhance the sophistication of our representation of hazard events. These models must be easy to use and adaptable to changing local needs by users. Unfortunately, many of the hazard models are too complex to be used by emergency managers and may not be adapted for unique local geographic, atmospheric or geologic conditions. Research by both the academic and military agencies will result in continuing developments in hazard models.

\section{Data Resources}

The collection of data relating to disasters is critical in emergency planning, response, recovery, and mitigation efforts. Data relating to the actual or potential disaster allows the emergency manager to understand the pheromone, whether it is a natural occurrence or man made as in an accidental chemical release of hazardous materials. Data also allows the emergency management community to use real data to simulate potential disasters as in the case of floods, a storm surge, earthquakes, or wind. Models have been developed to allow for the simulation of a disaster. These simulations require specific data concerning the natural or man made circumstances, the weather conditions, terrain, or physical structures.

Computer models that simulate natural or man made events require a variety of data sets. As an example, flood-modeling programs require information on the type of soil, land use characteristics, and elevation points in the study area. Remote sensing technologies provide precise definition of ground elevations, surface features (type of land cover) and weather conditions. In many cases, the data is very accurate and meets engineering quality standards. Direct access to data sources is utilized in many complex hazard models.

Research in real time or more accurate weather data, chemical references and digital libraries, chemical inventories, accidental release data, land contours, and population data will be critical to efforts to model hazards and examine their social, economic, and environmental impacts. Computerized sensors allow us today to see more clearly than 
ever before from aircraft as well as satellites; the sensors also allow us to understand the nature of the natural environment including winds, soil types, terrain, water movement, or vegetation. The following section provides an overview of direct and remote sensing data systems and their use in emergency management. New developments in direct and remote sensing data such as the USGS River Gauge System and the MODIS satellite support both research and operational decisionmaking. The application of high-resolution LIDAR land data to flood and storm surge modeling greatly enhances current hazard modeling efforts. Research to characterize hazard and their impacts must be continued to enhance the emergency management system.

Access to research data and studies by way of the Internet make information access timely and up to date. The Internet facilitates the communication of information between users by means of digital transmission technology. The Internet has been recognized for the potential of communicating information about emergencies and disasters. The Internet has been given credit for the facilitation of new partnerships and new models of organization for management. For developing countries, the Internet promotes intercountry collaboration on disasters by allowing quick and easy follow-up on commitments made during face to face meetings of country disaster managers; provides a low cost approach for exchanging expert information on specialized topics of importance during disasters; and allows researchers and practitioners in developing countries to access computerized databases and scientific literature available in developed countries (PAHO 1996).

\section{Operational Issues}

Stanley and Waugh (1999) suggest that the future of state and local government in the U.S. will be shaped by a combination of forces, including technology, politics, economics, demographics and environmental change. Public agencies at all levels will need to understand the implications of establishing partnerships with the private and not for profit sectors to maximize the greater potential of resource utilization within the community. Research must examine how the emergency manager can be the catalyst to eliminate the incongruities between government agencies, operating not in isolation but in relation to all other departments and agencies within government (Stanley and Waugh 1999).

\section{Linking the Research Community and Emergency Management Agencies}

The basic function of management is to align not only people, but also the institution itself including technology, processes, and structure. It attempts to reduce uncertainty at the same time searching for flexibility. Champy (1996) in Reengineering Management suggests that organizations face a significant barrier in attempting to bring change to the organization. Reengineering occurs when we introduce new technologies that alter the system. New technologies may require new configurations that must be defined. The introduction of new technologies provides the research community with an opportunity to reassess the emergency management process including the role of 
technology. The emergency manager must work hand-in-hand with the research community so that a full understanding of the implications of the change can be understood. Although Champy was not thinking just of emergency management or technology, he was stating that the manager must view the introduction of technology as a change in the system. Therefore, the manager should examine the impact that the technology will have on the current system and how this change can be implemented effectively.

Emergency management is a human system that utilizes technology. Technology needs to be easy to use for anyone in the emergency management process. It should not be viewed as an "expert system" only available to a select few. Ongoing training for officials will be critical in the effective use of technology in crisis situations.

\section{Using Technology to Create Synergy}

The combined and coordinated actions of the parts of the emergency management system achieve more than all of the parts acting independently. This approach suggests that if the performance of an enterprise is a product of the interaction rather than sum of its parts, it is entirely possible for the action of two or more parts to achieve an effect of which either is individually incapable (synergy). It explains why the performance of a system as a whole depends more on how its parts relate than on how well each part operates. Indeed, the inter-dependence of the parts is such that even if each part independently performs as efficiently as possible, the system as a whole may not. Technology is a critical element in establishing synergy in the emergency management system. Technology can facilitate or inhibit communication and cooperation within the system. Additional research is needed that examines how technology impacts the partnerships between the public, private, and non-profit emergency management system.

\section{Looking for the Quick Fix}

Technology can bring to the emergency management community an intelligent assistant for enhancing decision-making. It is not intended to replace individual discretion but serve as a tool for analysis and decisions. The technology revolution has resulted in more than just innovation, but also adjustments in social interactions. The increase in technology, especially communications, provides more information for decision-making; this should be a positive impact if it were not for inaccurate, incomplete or misdirected information. So often "technological developments lead to the notion that there can be a technological fix for whatever the problems are (Quarantelli, 1997). Unfortunately the users of technology are looking for the quick fix, assuming that what is high technology will address their problem. Some suggest that this is a focus on "gadgetry" rather than a true understanding of the technology including its strengths and limitations. Information clarifies some issues but it obscures and complexities others. Today, unprecedented amounts of information can be brought to bear on issues of policy and action but the person who must use the information to make the decisions may become overloaded. Information overload and an interest by emergency management to use the new fad, 
suggests that one must remember that the human element in managing disasters must be preserved.

\section{The Role of the Emergency Manager}

Research in new applications of technology should not be implemented in isolation. Close ties between the research community and local, state and federal emergency managers should be established throughout the development process. This will ensure that the technology will be an asset and improve the emergency management process. The emergency manager needs to get involved in planning for the development of the use of technology. The application of geographic information systems (GIS) provides a good example. The manager must facilitate realistic expectations from technical staff, users, and other support staff who might assist in the development of GIS data. GIS development is a process of technological innovation and requires management attention appropriate to this type of activity. It should be active as opposed to passive management involvement in the project (Manager's Overview 1997). Failures of GIS in the past resulted not from technical difficulties but from a lack of realistic expectations of all parties associated with the project including GIS technicians, potential users, managers, and officials. Research can demonstrate that the process of integrating technology into the system is a vital part of enhancing emergency management.

GIS belongs to the class of computer systems that require the building of large databases before they become useful. Unlike many other computer applications where a user can begin use after the purchase of the hardware and software, the use of a GIS (as illustrated by FEMA's HAZUS MH modeling and consequence assessment program illustrates) requires that large spatial databases be created, appropriate hardware and software be purchased, applications be developed, and all components be installed, integrated and tested before users can begin to use the GIS. A complex and difficult task extensive planning is required. Problems often occur because:

- The GIS is not integrated with other systems where interaction may be desired;

- Staff do not fully understand the technology;

- Development time estimates differ from actual task time;

- GIS involves greater cost uncertainty;

- A greater likelihood exists that programmatic changes (to other parts of the emergency management system) will be needed during the development phases.

The significant management point here is that these are normal conditions in the adoption of a new technology. Management needs to anticipate that such events will happen, and when they do, take appropriate management action.

\section{Data Access and Use}

Critical information is now more available to emergency managers as a result of our expanding networks. Some sensors provide real time access to data. As an illustration, 
the U. S. Geological Survey has installed a system of stream monitoring gauges to provide timely information on water levels in streams and rivers. Sensors are installed in the streams and rivers and available over networks remotely to the emergency manager. More widespread use of sensors will allow emergency managers greater access to valuable inputs for decision support systems.

The increasing attention to technology may inadvertently result in information overload. If a communication system is highly centralized, even a limited emergency can result in communication bottlenecks. Sub-networks may need to be created to divert communication from the operations center. A planned distributed computing capability can be designed by examining elements of the emergency management system. Who needs to communicate directly and frequently with whom?

Moving from centralized to decentralized systems may result in data integration problems. Many organizations have been struggling to cope with increasing demands for timely and accurate data to support ongoing decision-making. As a result, large centralized systems have been adapted resulting in more distributed databases. Maintaining linkages and integration of the data is critical in times of crisis. In the future, data will include more databases (relational), but also digital libraries, and multimedia databases maintained in a distributed environment. Documentation on the source of data and data types will be critical to ensure that information is used for its intended purpose.

The development of more powerful computers provides access to real time response resources. In a disaster, complex computing may not be possible for those directly involved in the response. As in other disaster management situations, a rear support team can be organized to carry out high performance modeling and analysis. Dependable networks should be designed to allow for detailed analysis to occur even during very stressful events. Linkages between support staff and operations units must be maintained in a secure environment.

\section{Recommendations on the Research Process}

The Report Computing and Communications in the Extreme: Research for Crisis Management and Other Applications by the National Academy Press made several recommendations that are appropriate in an examination of research in technology and emergency management.

\section{Develop User Centered Systems}

Ease of use by the emergency management community is the key. Each application must fit a specific need and where possible be integrated into other emergency management functions. Input by a variety of users is critical to ensuring that the application fits current and emerging problems. As a result, a broader interdisciplinary team may need to test the system to ensure its successful application. The current FEMA Hazards United States Multi-Hazards (HAZUS-MH) program requires expertise 
from many local agencies. Few agencies will likely have the capacity to use this complex planning and response tool without the support and involvement of other agencies. This facilitates a broader understanding of the system and encourages collaboration. In addition, training of staff in the use of the system is fundamental to ensure successful response. Research must support the development and implementation of interdisciplinary efforts to train a new generation of emergency management professionals. The HAZUS-MH program is an excellent example of technology that is user focused and may be applied to different hazards, geographic scales, and types of vulnerability analysis.

\section{Use Customized and Off the Shelf Programs}

Many third party software applications are designed to address a single emergency management function such as weather forecasts, emergency alert, or the maintenance of a database. The result is an application that is not integrated with other applications. Initially, integrated emergency management software may initially have a higher cost, but may be balanced with greater assurances of upgrades, maintenance, training, and user acceptance. Integrated software could be more cost effective in the long run and offer greater capability to link to other agencies in the emergency management system.

\section{Take into Account Human Factors}

Technology should not be viewed as an "expert system" only available to a select few, but easy to use by all those associated with the emergency management system. Orientation and training for new applications will be critical to ensure that the system will function.

\section{Take into Account Organizational Factors}

Public, private and non-profit emergency management agencies function within larger systems. Each organization faces financial limitations on expenditures for technology; purchases must be viewed as cost effective. Agencies must also be able to function seamlessly within their system and with larger local and national systems. Networking will enable agencies to communicate seamlessly but with safeguards.

\section{Conclusion}

The application of new technologies in emergency management requires ongoing training and education. To say the least, those in the emergency management must be willing to continue to learn and keep up to date. The digital environment of the Internet is an excellent means of making information available to a broader community in a timely manner. Digital libraries can go well beyond just the preservation and dissemination of knowledge, but also as an active partner with the potential to stimulate, support and register the process of creation of that knowledge. Methods of distance learning provide an active means of acquiring knowledge and skills from different perspectives or raising complex questions that can lead to answers. Creating and 
maintaining a learning environment thus plays a critical role in opening organizations to new technologies and their application to emergency management (Gladney et al., 1994). Research must support the development and implementation of interdisciplinary efforts to train a new generation of emergency management professionals. While we work to bring new technology to traditional emergency management agencies, initiatives should be taken to integrate other public, private and non-profit agencies in the emergency management process. Enhancing partnerships with other agencies allows for a broader approach to addressing emergency management issues.

\section{References}

"Application Needs for Computing and Communications (Chapter 1)," Computing and Communications in the Extreme: Research for Crisis Management and Other Applications. Workshop Series on High Performance Computing and Communications, Computer Science and Telecommunications Board, National Research Council. ht tp://www. nap.edu/readingroom/books/extreme/

Champy, James. Reengineering Management. New York: Harper Business, 1995.

Drager, K. Harald, Gunnar G. Lovas, J. Wiklund and Helge Soma. "Objectives of Modeling Evacuation from Buildings During Accidents: Some Path-Model Scenarios,” Journal of Contingencies and Crisis Management. Vol. 1 \#4, Dec. 1993.

"Manager's Overview, Needs Assessment and Conceptual Design of the GIS," Helping Local Government Create a GIS (Volumes 1), New York State Archives and Records Administration, National Center for Geographical Information and Analysis - State University of New York at Buffalo and GIS Resource Group, Inc. 1997, pp. 1 - 44). www.geog.buffalo.edu/ncgia/sara (Volumes 2 and 3 are suggested as references.) (See Readings: 4-Volume1.prf for a copy of this document. Volumes 2 and 3 are also available.)

PAHO (Pan American Health Organization). 1996. "The Internet: Tending to the Basics.” Disasters: Preparedness and Mitigation in the Americas 65: 1,7.

Quarantelli, E. L. "Problematical aspects of the information / communication revolution for disaster planning and research: ten non-technical issues and questions." Disaster Prevention and Management. Vol. 6 \#1, 1997.

Stalberg, Christian E. " The Intelligent City and Emergency Management in the 21st Century," Proceedings: The International Emergency Management and Engineering Conference, April 18-21, 1994. (See Readings: 1-Stalberg Word Document)

Stanley, Ellis M. Sr., and William L. Waugh, Jr. "Emergency Managers for the New Millennium," Handbook of Crisis and Emergency Management. Edited by Ali Farazmand. New York: Marcei Dekker, 1999.

Wahl, Kenneth L., Wilbert O. Thomas, Jr., and Robert M. Hirsch. "Stream-Gauging 
Program of the U.S. Geological Survey,” U.S. GEOLOGICAL SURVEY CIRCULAR 1123, Reston, Virginia, 1995, http://water.usgs.gov/public/realtime.html 